\title{
BMJ Open Quality Improving local anaesthetic systemic toxicity (LAST) awareness in maternity care using tailored educational tools
}

\author{
Amy E Edwards, ${ }^{1}$ Gemma M Bowsher, ${ }^{1}$ Sahil Deepak, ${ }^{1}$ Mohamed $A i^{2}$
}

To cite: Edwards AE, Bowsher GM, Deepak S, et al. Improving local anaesthetic systemic toxicity (LAST) awareness in maternity care using tailored educational tools.BMJ Open Quality 2018;7:e000070. doi:10.1136/ bmjoq-2017-000070

Received 27 March 2017 Revised 26 February 2018 Accepted 27 February 2018
Check for updates

${ }^{1}$ GKT School of Medical Education, Faculty of Life Sciences \& Medicine, King's College London, London, UK ${ }^{2}$ Department of Anaesthetics, Kent \& Canterbury Hospital, East Kent Hospitals University NHS Foundation Trust, Canterbury, UK

Correspondence to

Amy E Edwards;

amy.a.edwards@kcl.ac.uk

\section{ABSTRACT}

Local anaesthetic (LA) agents are widely used in maternity care. Although relatively safe, their use does carry risks, the most serious of which is systemic toxicity (LAST). LAST poses a major threat to maternal and neonatal safety due to the frequency of LA administration in maternity care and the under-recognition of toxicity in such settings, which has been reported globally. Our aim was to prevent LAST occurrence in a District General Hospital (DGH) maternity unit by improving staff awareness through the implementation of a tailored educational programme. We used a standardised 14-point questionnaire to evaluate LAST awareness among staff of all disciplines. Domains of interest were LA maximum safe doses, LAST recognition, immediate management and use of antidote. Following baseline assessment, we implemented an educational programme in three stages. Each featured a distinct tool: video presentation, poster and lanyard card. Awareness was reassessed between stages using the same questionnaire. We identified poor baseline awareness across all non-anaesthetic disciplines. Average questionnaire score improved from 3.9/14 $(n=23)$ to $8.1 / 14(n=30)$ during the project period, an increase of $109.3 \%$. Scores improved in all professional groups and a change in workplace culture has been reported. Using a tailored interprofessional educational intervention, we generated an increase in awareness and maintained this over a 4-month period. Improved knowledge and a shift in clinical attitudes towards shared responsibility will reduce avoidable peripartum risk associated with LAST at this DGH. Although the tools used were specific to LAST in this setting, they could be easily adapted for NHS maternity services elsewhere and indeed other areas of care.

\section{PROBLEM}

Queen Elizabeth The Queen Mother hospital (QEQM), a DGH in Margate, is one of two hospitals within East Kent Hospitals University NHS Foundation Trust (EKHUFT) to provide full maternity care: both midwifery and obstetrician-led services. It serves a large geographical area and diverse population in east Kent, and has approximately 2800 births every year. ${ }^{1}$

We observed inconsistent communication of LA risks in this unit, which we suspected were due to poor knowledge as had been reported elsewhere. ${ }^{2-4}$ Conversation with maternity staff suggested that many were unfamiliar with LAST and there was confusion with anaphylaxis. As baseline data collection confirmed poor LAST awareness across disciplines, and as an interprofessional approach to Quality Improvement (QI) has been associated with maximal safety benefit, ${ }^{5}$ we were confident that we could expect to improve clinical awareness during this QI project.

\section{BACKGROUND}

LAST is the most serious avoidable risk associated with LA administration and more common than assumed, occurring in up to 1/1000 peripheral blocks. ${ }^{6}$ Furthermore, LAST is believed to be under-recognised and reported and thus incidence underestimated. ${ }^{7}$ This represents substantial risk in maternity settings due to the frequency of LA usage. It is therefore critical that such risks are well understood and communicated, and all staff are equipped to manage complications. ${ }^{8}$ Previous studies have sought to evaluate awareness of $\operatorname{LAST}^{7}$; however, there have been no documented attempts to address this lack of awareness through formal QI intervention.

National guidelines for the recognition and management of severe LAST exist, published by the Association of Anaesthetists of Great Britain and Ireland (AAGBI) in 2007. ${ }^{9}$ EKHUFT endorses these and they can be accessed through local intranet pages. Through informal interviews with staff at the time of baseline data collection, we revealed widespread belief among maternity unit staff that the guidelines were intended for and only relevant to anaesthetists, on account of their being published by that professional body. This highlighted the problem that LAST was not perceived as something for which responsibility was shared between disciplines, lending further support to our use of an interdisciplinary approach. 


\section{MEASUREMENT}

Describe which measures you selected for studying processes and the outcomes of the intervention(s), including rationale for choosing them, their operational definitions and their validity and reliability. Describe how you planned to collect these data throughout your project and how frequently. Outline how you planned to establish if the observed outcomes were due to your interventions.

You must also include here, the results of your baseline measurement. There is a good example in this project entitled 'Improving communication between phlebotomists and doctors: a quality improvement project'.

We chose to investigate LAST awareness among staff using a questionnaire, as this would enable rapid and repeatable data collection with minimal disruption to the usual running of the unit. LAST awareness had recently been investigated as part of an audit in the surgical department of another hospital within the trust and the use of a questionnaire was successful. We modified this questionnaire for application in the maternity unit.

The questionnaire comprised qualitative and quantitative questions. Qualitative questions aimed to ascertain the general understanding of LAST and any previous experience of such events. Some of these questions required 'yes/no' answers, for example, "Have you ever encountered LAST in your work?" and some involved free-text responses. Quantitative questions aimed to further explore LAST awareness in four key domains and provide a scoring system, which could be used to measure change over time, for example, correct selection of three clinical features of LAST from a list. Where possible, multiple choices were offered for question responses. The four domains of interest were LA maximum safe doses, signs and symptoms (recognition) of LAST, immediate management of LAST and the use of lipid emulsion as antidote, both in theory and in practice (site-specific). The maximum score available for these questions was 14 .

The same questionnaire was to be redistributed following each intervention. The only change to be made was the addition of a qualitative question to find out if each respondent had been aware of or used the educational tools provided. Thus, we would be able to determine whether changes observed were attributable to our interventions. Due to the nature of questionnaire dissemination, it was not possible to calculate response rates; paper questionnaires were distributed in workspaces and links to online formats shared in departmental communication. Responses were gathered from all professional groups regularly working in the unit: midwives (bands 5-8), maternity care assistants, nurses, student midwives, medical students, non-specialist and specialist junior doctors, consultant anaesthetists and obstetricians. The majority of respondents were midwives.

Baseline questionnaire responses revealed poor awareness of LAST across all domains with a mean total score of 3.87/14 $(\mathrm{n}=23)$. Scores were poor in all professional groups except anaesthetists; however, it was observed that even these specialists were not scoring maximally.
We were also made aware that non-anaesthetists had not previously received any teaching relating to LAST.

We set our target improvement to $50 \%$ as although we were confident that greater improvements would be possible, we also recognised factors that were likely to limit the impact of our educational programme, including the short timeframe, high staff turnover and limited face-toface input from us.

\section{DESIGN}

Describe the intervention (or series of interventions) that you planned to implement to improve the quality of care you deliver. Describe any reasons or assumptions that were used to develop the intervention(s) and reasons why you expected them to work. Outline how you consulted/ engaged with your team members or organisations. Who was in your project team? Did you anticipate/predict any problems at this stage? Describe how you planned to make your intervention sustainable.

This step is critical for others to understand the thinking behind the development of your intervention.

There is an example in this article entitled 'Improving asthma severity and control screening in a primary care pediatric practice'.

Identification of poor LAST awareness and a paucity of previous teaching on the topic across disciplines confirmed that there was scope for substantial improvement with educational intervention. A change in perception of LAST was also required to promote cross-disciplinary responsibility for its active prevention.

We designed an interprofessional education programme according to QI methodology outlined by the Institute for Healthcare Improvement, using distinct tools for each of three 'plan-do-study-act' (PDSA) cycles. Each PDSA cycle was 4 weeks in duration, involving a 2-week period for educational tool impact and a 2-week period for subsequent data collection. We involved staff groups by empowering key stakeholders (including the midwifery matron and obstetric anaesthetists) to supervise the project themselves.

This project was exempt from ethics review.

\section{STRATEGY}

\section{PDSA 1: educational video presentation}

This was designed to have broad impact as baseline data revealed poor scores across all four domains of awareness (LA safe maximum doses, recognition, initial management and antidote details) in all staff groups except anaesthetists. We created a PowerPoint presentation that provided comprehensive but concise information on LAST, suitable for all disciplines. Delivering this via prerecorded video ensured that all staff would be able to access the information flexibly, irrespective of work schedule. We predicted that improvements in all domains would be seen across disciplines. 


\section{PDSA 2: educational posters}

This was designed to reinforce information introduced in the video presentation. However, we became aware through informal interviews that as midwives do not directly administer or prescribe LA, information regarding safe doses was not relevant to their educational needs and thus not suitable for inclusion in a pan-disciplinary educational programme. Additionally, this information might deter some staff from engaging fully by seeming irrelevant or intimidating. As such, safe doses were excluded from posters. All other information was included: signs and symptoms, tips on LAST recognition, appropriate management and location of lipid emulsion in the workplace. We predicted improvements in recognition, initial management and antidote awareness, and for the greatest improvements to be among midwives as they were permanently based in areas where posters would be displayed.

\section{PDSA 3: lanyard card}

This was designed to complement the posters, highlighting key points and serving as a reminder to refer to them. We predicted similar (further) improvements to those expected following intervention 2.

Although interventions were planned before their introduction, specific areas for improvement were identified between cycles based on questionnaire responses and staff feedback. Details of subsequent interventions adjusted accordingly in order to maximise potential impact and benefit.

\section{RESULTS}

Baseline questionnaire responses revealed poor awareness of LAST across all domains with an average total score of 3.87/14 ( $\mathrm{n}=23)$. As shown in our run chart (Figure 1), we were successful in generating an improvement in average questionnaire score. Above-target improvement was achieved after PDSA 1 and maintained throughout subsequent cycles.

Data collected at the end of PDSA 1, following the introduction of our educational video, revealed an improvement in average score to $6.57 / 14(\mathrm{n}=21)$. This represents a $69.8 \%$ improvement from baseline score by the end of December, and the greatest increase in score seen following a single intervention (in one PDSA cycle). On further interrogation of our data, we found clear improvement across the four target domains and in all professional groups. This included consultant anaesthetists, achieving a $27.3 \%$ improvement, and student midwives, achieving at $91.7 \%$ improvement. We are confident that these increases are attributable to our interventions as all respondents in this cycle reported having viewed our video during the preceding fortnight.

Data collected at the end of PDSA 2, following the introduction of the posters, demonstrated an improvement in average score to 8.96/14 $(\mathrm{n}=22)$. This represents a further $30.3 \%$ improvement in average score after PDSA 1 , and a $130.7 \%$ improvement from baseline by the end of January. As this increase is also considerable and as the majority of respondents in this cycle reported having seen

\section{Average LAST Awareness Score in Maternity Care Unit at QEQM}

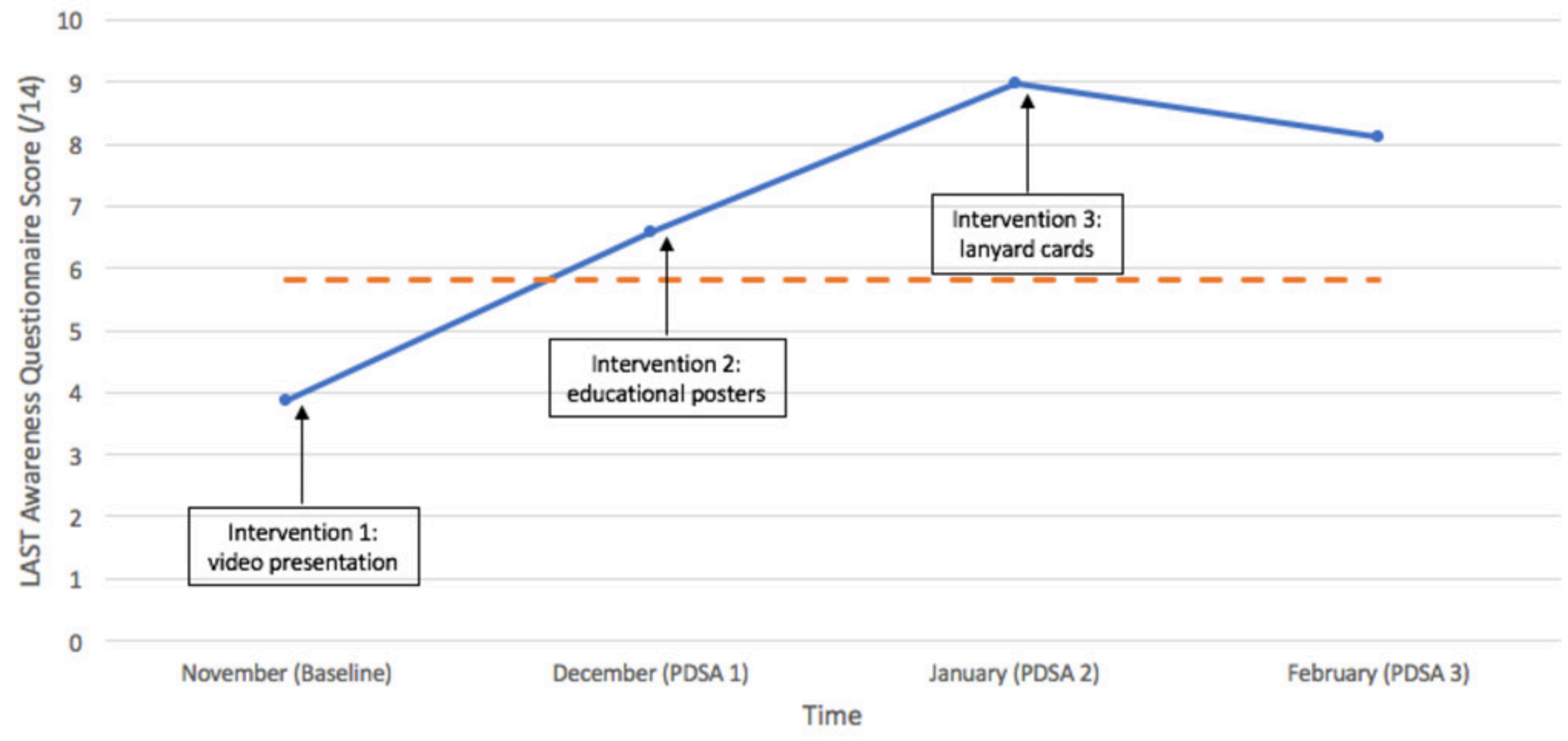

Figure 1 Average local anaesthetic systemic toxicity (LAST) awareness score over time; run chart. Blue points indicate plando-study-act (PDSA) data collection: average scores after each intervention (described in boxes). 50\% target improvement from baseline is shown in orange. 
our posters, it is reasonable to conclude that the reinforcement of prior learning using complementary visual media is an effective strategy in maintaining and further enhancing that learning.

Data collected at the end of PDSA 3, following distribution of lanyard cards, showed an average score of $8.1 / 14(n=30)$. This represents a reduction of $9.6 \%$ from average score after PDSA 2, however an overall improvement from average baseline score of $109.3 \%$ by the end of February. The slight reduction in score was not predicted and would suggest that lanyard cards were not effective in maintaining the improvements in awareness that we had achieved. This could perhaps be because such tools are only likely to be accessed 'in the event', and events themselves are rare.

\section{LESSONS AND LIMITATIONS}

We demonstrated that LAST is not well understood, even among specialist care-providers, and is likely under-recognised. ${ }^{10}$ This important preventable cause of morbidity and mortality should be considered across the National Health Service (NHS) services. Raising awareness (and therefore recognition and management) of LAST is critical in preventing harmful events and associated financial implications for trusts. ${ }^{11}$

We used guidelines published by the $\mathrm{AAGBI}^{9}$ in designing our interventions to ensure accuracy of information. However, as these were not specific to the maternity setting and were intended for a specialist medical readership, we amended them to improve their relevance and accessibility to non-medical professionals. We also consulted professional guidelines for non-medical staff to encourage engagement by maximising career development incentives, ${ }^{12}$ and our clinical aim was informed by an NHS patient safety alert. ${ }^{8}$ This project demonstrates successful incorporation of guidelines into routine clinical practice.

As we were not permanently based at our project site, we needed to adapt interventions to ensure that we could make improvements remotely. Our presence was required for questionnaires to be completed, possibly due to inadequate staff incentive and professional cultures. ${ }^{1314}$ It seemed that there was suboptimal expectation of continual research and improvement, which we also felt that we had challenged with some success. The project was also completed without funding, ensuring that tools used were produced at low or no cost.

Aspects of this project key to its success included tailoring educational tools specifically to the needs of their recipients, and empowering staff by direct involvement in project design and delivery. This proved essential in maintaining engagement. Understanding the culture of a particular environment is also important in this respect, and finding the balance between being sympathetic to and challenging this is how change can be made effectively. We will now be able to apply these principles in our future practice and generate QI in other areas.

\section{CONCLUSION}

We have substantially raised awareness of LAST among maternity unit staff of all disciplines at QEQM, using a tailored educational programme. We surpassed our target and maintained improvements throughout the 4-month duration of the QI project.

We have demonstrated that it is possible to produce and maintain a significant improvement in awareness of this issue within a short timeframe, with minimal cost and for the most part, remotely. This contributes to improving patient safety at QEQM; however, as most of the information provided is neither specific to maternity care nor the site, our interventions could easily be applied across the NHS on a wider scale. In fact, we have already provided some resources to other sites within the trust and our video is now included in trust induction teaching for medical students on Women's Health (Obstetrics, Gynaecology and Sexual Health) rotation. Suggestions have been made to include this topic and our resources in annual Mandatory and Statutory Training requirements for unit staff, and for the nomination of a LAST champion in the maternity unit at QEQM.

By involving staff and students across all disciplines, we have also promoted a culture of shared responsibility for LA use in the unit. We received reports and indeed observed ourselves that senior midwives were discussing LAST with new student midwives and juniors, which may well be reflected in our data. Additionally, educational tools introduced as project interventions have become part of induction programmes for medical students and plans are being made to extend this to mandatory and statutory training of permanent staff. Both this cultural shift and the resources we have provided will survive well beyond our project and should continue to improve patient safety.

Contributors AEE, GMB and SD contributed equally to project design and implementation, under the supervision of MA. All authors contributed to the manuscript. AEE reviewed and submitted the manuscript.

Funding This research received no specific grant from any funding agency in the public, commercial or not-for-profit sectors.

Competing interests None declared.

Provenance and peer review Not commissioned; externally peer reviewed.

Open Access This is an Open Access article distributed in accordance with the Creative Commons Attribution Non Commercial (CC BY-NC 4.0) license, which permits others to distribute, remix, adapt, build upon this work non-commercially, and license their derivative works on different terms, provided the original work is properly cited and the use is non-commercial. See: http://creativecommons.org/ licenses/by-nc/4.0/

(C) Published by the BMJ Publishing Group Limited. For permission to use (where not already granted under a licence) please go to http://www.bmj.com/company/ products-services/rights-and-licensing/

\section{REFERENCES}

1. East Kent Hospitals University NHS Foundation Trust. Maternity services in East Kent. 2016. www.ekhuft.nhs.uk/patients-andvisitors/services/pregnancy-and-childbirth/

2. Karasu D, Yılmaz C, Özgünay ŞE, Ş Özgünay, et al. Knowledge of the research assistants regarding local anaesthetics and toxicity. Turk $J$ Anaesthesiol Reanim 2016;44:201-5. 
3. Sagir A, Goyal R. An assessment of the awareness of local anesthetic systemic toxicity among multi-specialty postgraduate residents. J Anesth 2015;29:299-302.

4. Jensen-Gadegaard P, Skjønnemand M, Damgaard-Jensen J, et al. Limited knowledge of lipid rescue therapy in local anaesthetic systemic toxicity. Dan Med Bull 2011;58:A4226.

5. Danielsson $\mathrm{M}$, Nilsen $\mathrm{P}$, Rutberg $\mathrm{H}$, et al. A national study of patient safety culture in hospitals in Sweden. J Patient Saf 2017:1.

6. van der Nest L. Local anaesthetic agent systemic toxicity. Continuing Medical Education 2012;30:215-6.

7. Collins J. Correspondence: awareness of local anaesthetic toxicity issues among hospital staff. Anaesthesia 2010;65:960-1.

8. NHS National Patient Safety Agency. Safer practice with epidural injection and infusions. Patient Safety Alert 2007 www.nrls.npsa. nhs.uk/EasySiteWeb/getresource.axd?AssetID=60063\&type=full\& servicetype=Attachment
9. Association of Anaesthetist of Great Britain and Ireland (AAGBI) Guidelines. Management of Severe Local Anaesthetic Toxicity. 2010. https://www.aagbi.org/sites/default/files/la_toxicity_2010_0.pdf

10. Collins $\mathrm{J}$. Awareness of local anaesthetic toxicity issues among hospital staff. Anaesthesia 2010;65:960-1.

11. Christie LE, Picard J, Weinberg GL. Local anaesthetic systemic toxicity. BJA Educ 2015;15:136-42.

12. Nursing and Midwifery Council. Professional code for Nurses and Midwives. 2015. www.nmc.org.uk/standards/code/read-the-codeonline

13. Reeves S, Perrier L, Goldman J, et al. Interprofessional education: effects on professional practice and healthcare outcomes (update). Cochrane Database Syst Rev 2013;4.

14. Hojat M, Fields SK, Rattner SL, et al. Attitudes toward physiciannurse alliance: comparisons of medical and nursing students. Acad Med 1997;72:S1-3. 